\section{Hypertophic pulmonary osteoarthropathy: a striking association with a malignant solitary fibrous tumour of the pleura}

\section{Hunt ${ }^{1} \quad$ B Barber ${ }^{2} \quad$ P Cane $^{3} \quad$ T Treasure $^{2}$}

J R Soc Med 2007; 100:427-428

Lung and chest wall tumours may present with joint pain. A chest $\mathrm{X}$ ray may reveal the cause.

\section{CASE HISTORY}

A 66-year-old man complained of right knee pain and was treated for arthritis in primary care for over a year. Analgesics had minimal effect. He lost weight, was found to have a raised C-reactive protein and his GP referred him to a rheumatologist. At this point he had no chest symptoms and an unremarkable past medical history. He was an exsmoker, having stopped five years previously. At his rheumatology appointment clubbing of both his fingers and toes was seen (Figure 1). The clinical and radiological features were of hypertrophic pulmonary osteoarthopathy (HPOA). Chest X-ray showed an opacity in the left chest (Figure 2), and computed tomography (CT) showed a rounded mass (Figure 3). These radiological features are of solitary pleural tumour for which surgical excision is the first line of treatment.

A CT-guided biopsy was reported to show an undifferentiated tumour so we first performed video assisted thoracoscopy (VATS) to assess the feasibility of completely removing it. We found that the tumour was completely free from the parietal pleura and mobile on the mediastinum, so we judged removal to be possible. We discussed in the thoracic cancer multidisciplinary meeting and the oncology team agreed surgical removal without induction chemotherapy. Complete surgical excision was performed and the diagnosis confirmed as benign solitary fibrous tumour. He recovered well and his previously unrelenting knee pain had disappeared the following day. At

${ }^{1}$ Department of Cardiothoracic Surgery, St George's Hospital, Blackshaw Road, London SW17 OQT, UK, Departments of ${ }^{2}$ Thoracic Surgery and ${ }^{3}$ Pathology, Guy's Hospital, London, UK

Correspondence to: Mr lan Hunt

Email: ianjhunt@gmail.co.uk

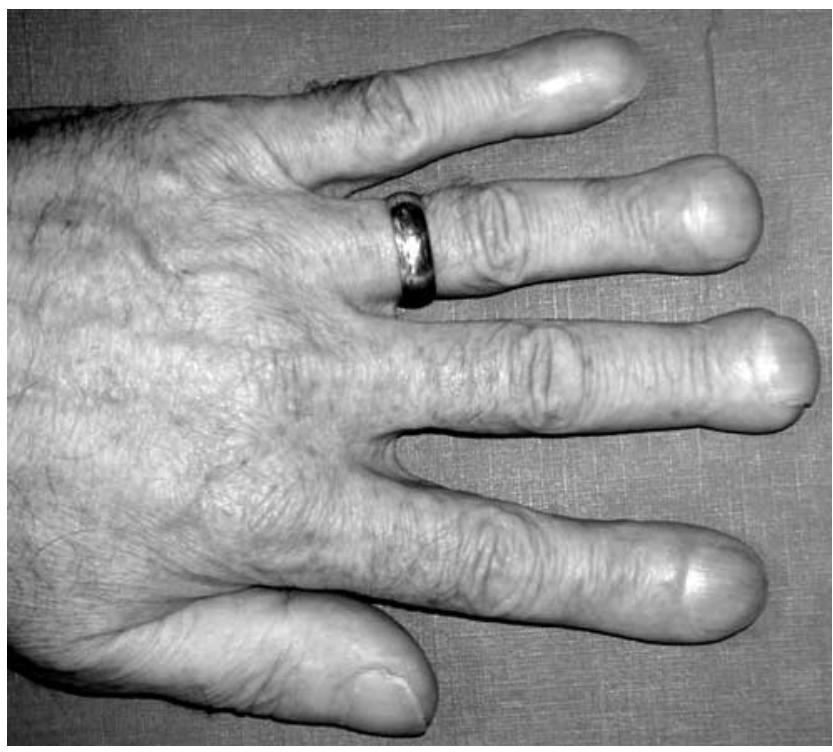

Figure 1 Severe clubbing of fingers prior to resection

six weeks he had no knee pain but the periostitis of the femur was still evident (Figure 4).

\section{DISCUSSION}

The combination of finger clubbing and a mass on chest $\mathrm{X}$ ray is a familiar presentation of lung cancer. Pleural disease may also be associated with clubbing and HPOA. ${ }^{1}$

Benign solitary fibrous tumours of the pleura are slow growing but can compress adjacent structures. They usually arise from the visceral pleura, and radiologically it can be difficult to tell if they are pleural or pulmonary in nature.

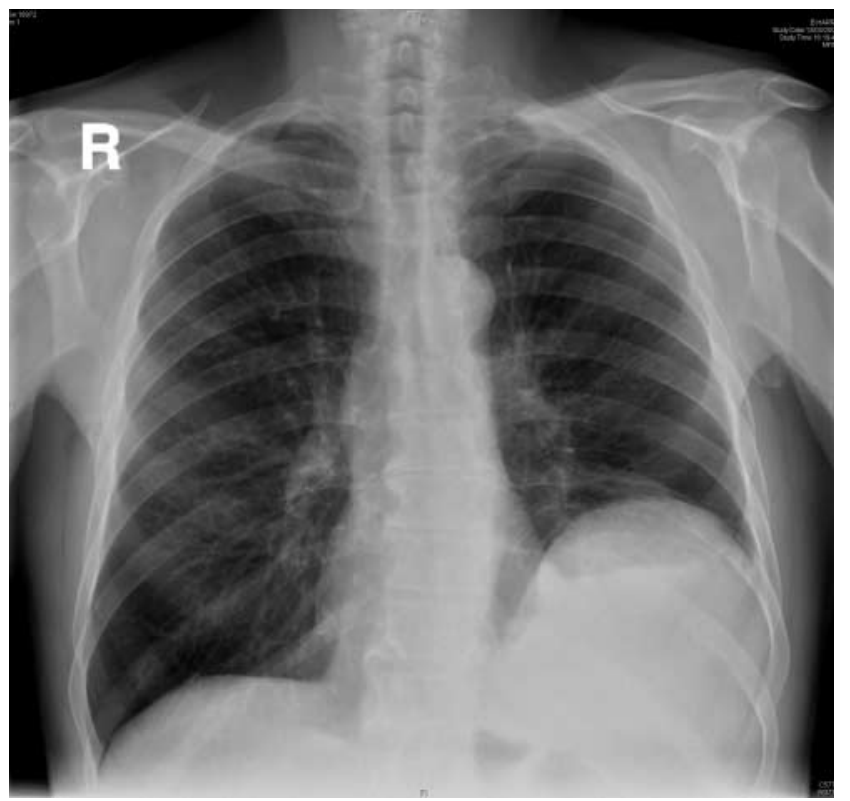

Figure 2 Chest $\mathbf{X}$ ray with elevation of left hemi-diaphragm 


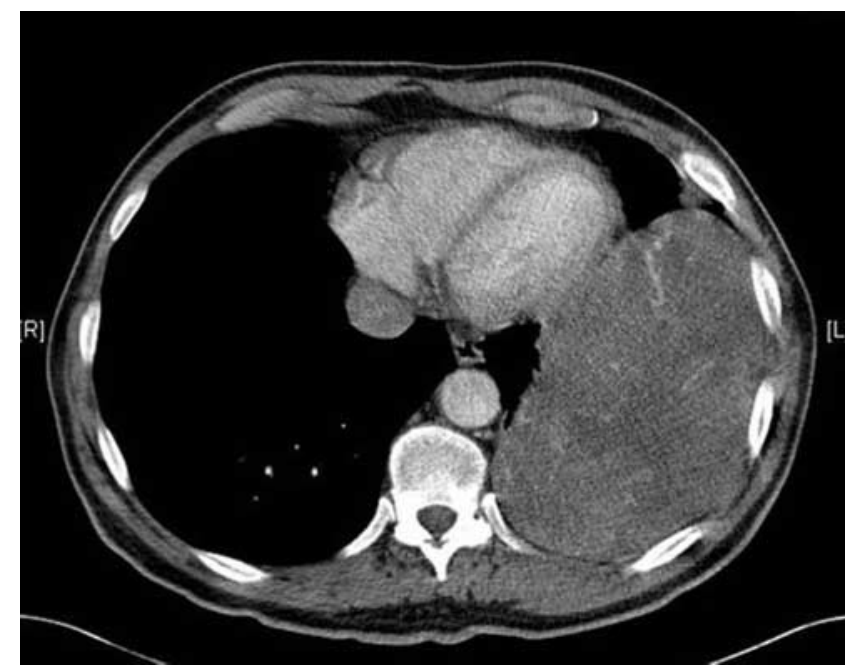

Figure 3 Chest CT showing tumour at the base of the left chest and related to the left lower lung or diaphragm

Histological diagnosis can be difficult from biopsies, as in this case, because the tumour may undergo partial infarction and lose its characteristic features. The diagnosis is based on the presence of bland spindles mixed with 'ropey' collagen juxtaposed by more cellular areas. The spindles cells characteristically stain for the immunohistochemistry marker CD34. Fewer than $20 \%$ of solitary fibrous tumours are malignant. Malignancy is suggested histologically by the presence of pleomorphic cells with atypical nuclei, high mitotic rate and necrosis. ${ }^{2}$

Pleural tumours as typified by malignant pleural mesothelioma grow as sheets, resembling diffuse pleural thickening; solitary fibrous tumours of the pleura form a near spherical mass. Unlike mesothelioma these tumours are of mesenchymal origin, not related to asbestos exposure and are usually benign. In describing these tumours, previous terminology has included benign mesothelioma, localized mesothelioma and localized fibrous tumour of the pleura. ${ }^{3}$ The variety of descriptive names may reflect the typical clinical course and difficulties in pathological typing due to the paucity of distinct histological features. Features associated with paraneoplastic syndromes, most noticeably

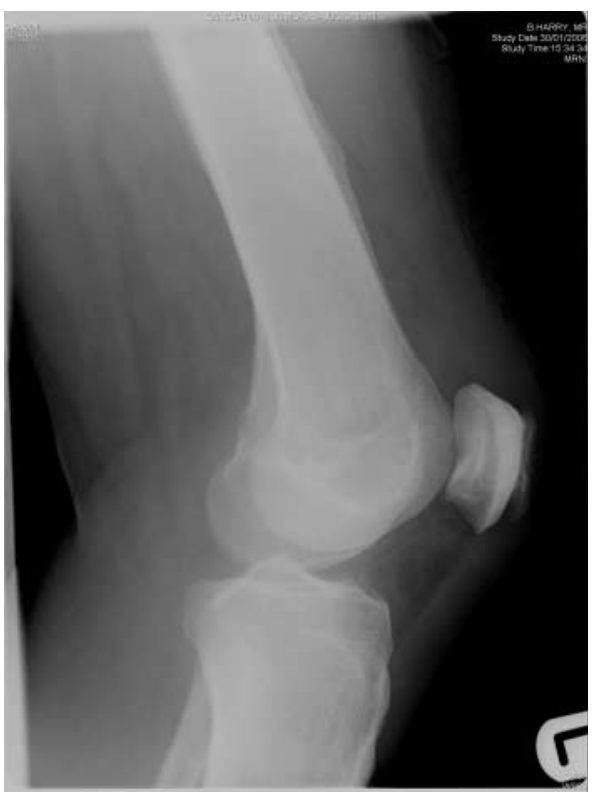

Figure 4 Lateral knee $\mathbf{X}$ ray demonstrating periostitis involving the femur still present following excision of the tumour

clubbing, hypertrophic osteoarthropathy and hypoglycaemia, are well described. ${ }^{2,4}$

Surgical resection of the tumour is the treatment of choice, with any associated paraneoplastic syndromes typically resolving following complete excision. ${ }^{5}$ Local recurrence is possible if incompletely excised, and malignant transformation has been reported. ${ }^{2}$ Long-term clinical follow up is recommended.

\section{REFERENCES}

1 McGavin C, Hughes P. Finger clubbing in malignant mesothelioma and benign asbestos pleural disease. Resp Med 1998;92:691-2

2 Kanthan R, Torkian B. Recurrent solitary fibrous tumor of the pleura with malignant transformation. Arch Pathol Lab Med 2004;128:460-2

3 Okike N, Bernatz PE, Woolner LB. Localized mesothelioma of the pleura: benign and malignant variants. J Thorac Cardiovasc Surg 1978;75: 363-72

4 Urschel JD, Anderson TM, Whooley BP. Finger clubbing and a lung mass. Chest 1999;115:1735-7

5 Treasure T. Hypertrophic pulmonary osteoarthropathy and the vagus nerve: an historical note. J R Soc Med 2006;99:388-90 